\title{
Evaluación del desarrollo positivo en adolescentes mexicanos: estudio exploratório
}

\author{
Assessment of positive development in Mexican adolescents: exploratory study \\ Diana Betancourt Ocampo ${ }^{1}$ \\ Alejandro González-González ${ }^{2}$ \\ María José Acedo Trueba ${ }^{3}$ \\ Celia Shamosh Hamui ${ }^{4}$ \\ Claudia Andrea Greene Torres ${ }^{5}$ \\ Samuel Ohrenstein Nasielskier ${ }^{6}$ \\ 123456 Facultad de Psicología, Universidad Anáhuac. México
}

\begin{abstract}
Resumen: El objetivo del presente estudio fue diseñar y valorar psicométricamente un instrumento que evalúa el desarrollo positivo de adolescentes mexicanos. Para lo cual se seleccionó una muestra no probabilística de 400 adolescentes, estudiantes de nivel secundario, 57\% fueron hombres y $43 \%$ mujeres, con un promedio de edad de 13.5 años. Con base en la revisión de la literatura se diseñó un instrumento basado en la propuesta de Lerner (2004), de 59 reactivos que se agrupan en cinco escalas Likert que evalúan las cinco características del desarrollo positivo: Competencia (13 reactivos, $\alpha=.809$ ), Cuidado ( 6 reactivos, $\alpha=.672$ ), Confianza (11 reactivos, $\alpha=.837$ ), Conexión (17 reactivos, $\alpha=.920$ ) y Carácter (12 reactivos, $\alpha=.854$ ). Los resultados mostraron que el instrumento cuenta con propiedades psicométricas adecuadas para este estudio. La discusión analiza la utilidad del instrumento en población adolescente mexicana para la valoración del desarrollo positivo y como instrumento para evaluar la efectividad de programas de promoción del desarrollo saludable en adolescentes.
\end{abstract}

Palabras clave: desarrollo positivo, adolescentes, evaluación psicológica, medición, escala

\begin{abstract}
The objective of this study was to design and measure psychometrically an instrument that assesses the positive development of Mexican adolescents. To this end, a non-probabilistic sample of 400 adolescents, selected from secondary school students, $57 \%$ were men and $43 \%$ were women, with an average age of 13.52 years. Based on the review of the literature, an instrument was designed based on the proposal of Lerner (2004), which comprises 59 items that are grouped into five scales Likert type that evaluate the five characteristics of positive development: Competence (13 items, $\alpha=.809$ ), Care (6 items, $\alpha=.672$ ), Confidence (11 items, $\alpha=.837$ ), Connection ( 17 items, $\alpha=.920$ ) and Character (12 items, $\alpha=.854$ ). The results showed that the instrument has adequate psychometric properties for this study. The discussion analyzes the utility of the instrument in the Mexican adolescent population for the assessment of positive development and as an instrument to evaluate the effectiveness of programs promoting healthy development in adolescents.
\end{abstract}

Keywords: positive development, adolescents, psychological assessment, measurement, scale

Cómo citar este artículo:

Betancourt Ocampo, D., González-González, A., Acedo Trueba, M. J., Shamosh Hamui, C., Greene Torres, C. A., \& Ohrenstein Nasielskier, S. (2018). Evaluación del desarrollo positivo en adolescentes mexicanos: estudio exploratório. Ciencias Psicológicas, 12(2), 261-269. doi: https://doi.org/10.22235/cp.v12i2.1690. 


\section{Introducción}

El Desarrollo Positivo de los Adolescentes (Positive Youth Development, PYD, ser más conocido por sus siglas en inglés) surge como una nueva perspectiva para el estudio de la adolescencia, la cual, tradicionalmente se enfocaba en comprender a este periodo de la vida como un periodo caótico y en donde los adolescentes se involucran en conductas que ponen en riesgo su salud. El PYD busca identificar factores del desarrollo particularmente importantes para predecir resultados favorables tanto físicos como emocionales y sociales (Lerner et al., 2005).

Heck y Subramaniam (2009) realizaron una revisión de los modelos más utilizados desde el PYD: 1) Cuatro Elementos Esenciales del Desarrollo, 2) Habilidades para la Vida, 3) Fortalezas del Desarrollo, 4) Enfoque de Acción Comunitaria, 5) Cinco características del desarrollo positivo (5 C's). Los primeros cuatro modelos, son propuestas que se enfocan en analizar aquellos factores (características, habilidades, fortalezas) que pudieran favorecer un desarrollo saludable en los jóvenes. El último modelo, se enfoca en analizar cuáles serían las características que definen a un joven con un desarrollo positivo (de ahí el nombre del modelo); de acuerdo con la revisión de estos autores, esta última propuesta es la que cuenta con mayor evidencia empírica.

Lerner $(2002 ; 2004)$ sugirió que las cinco características que describen a un adolescente con un desarrollo saludable son: Competencia (el dominio de ciertas habilidades intelectuales, sociales y comportamentales); Confianza (visión positiva de uno mismo, con un sentido de autoeficacia y voluntad propia); Conexión (lazos positivos con personas e instituciones); Carácter (integridad personal y moral asumida por uno mismo); Cuidado/ Compasión (valores humanos positivos, empatía y sentido de justicia social). Además, dentro de esta propuesta se considera que los jóvenes que incorporan estas cinco C's estarían en la posibilidad de demostrar una sexta $\mathrm{C}$ denominada Contribución, esto es, podrían contribuir a ellos mismos, a su familia, a su comunidad y a la sociedad civil; mientras que los jóvenes que posean poco de las cinco $\mathrm{C}^{\prime} \mathrm{s}$, podrían estar en riesgo de presentar problemas personales, sociales y conductuales (Heck \& Subramaniam, 2009; Lerner, 2004; Lerner et al., 2005).
El estudio del 4-H es un proyecto longitudinal que sirvió para que Lerner et al. (2005) diseñaran y evaluaran instrumentos que permitieran medir el modelo de las 5 C's. Este estudio surge del 4-H que es una organización juvenil de Estados Unidos que busca promover a través de diversos programas el bienestar en la juventud, y que de acuerdo con Lerner et al., (2005) podría ser un ejemplo de un programa desde la PYD. En este estudio longitudinal se realizaron mediciones desde el quinto grado (equivalente al último grado de primaria) hasta el décimo grado (equivalente a segundo grado de bachillerato). En la primera medición se diseñó y evaluó el primer instrumento para medir el PYD, el cual registraba información de dos informantes, por un lado el cuestionario que respondieron los padres o tutores (PQ, Parent Questionnaire, por sus siglas en inglés) y por otro, el cuestionario que respondían los estudiantes (SQ, Student Questionnaire, por sus siglas en inglés). Este último instrumento (SQ) comprendió aspectos tales como la evaluación de: 5 C's, problemas de conducta, nivel de desarrollo puberal, fortalezas individuales y ecológicas, regulación del desarrollo, actividades y aspectos demográficos. Cabe señalar, que para fines de la presente investigación nos enfocaremos en la medición de las 5 C's en la versión para estudiantes.

El instrumento de Lerner et al. (2005) que evalúa las 5 C's se conforma de 76 reactivos, cada una de las características evaluadas tienen diferente número de indicadores (ver tabla 1). Para el desarrollo de dicho instrumento, los autores eligieron una serie de instrumentos que ya se habían empleados en otros estudios y que permitían la valoración de las 5 C's. Por ejemplo, utilizaron el Profile of Student Life-Attitudes and Behaviors Surver (PSL-AB) desarrollado por Benson, Leffert, Scales y Blyth (1998) y Theokas et al. (2005) para evaluar las fortalezas (internas y externas) del desarrollo. Lerner et al. (2005) fundamenta el uso del $P S L-A B$ en tanto miden indicadores de las 5 C's. Algo que vale la pena mencionar, es que si bien, la mayoría de los indicadores utilizados cuentan con valores adecuados de confiabilidad (Bowers et al. 2010), esto no aplica para el compromiso escolar (indicador de competencia) donde de acuerdo con Jelicic, Bobek, Phelphs, Lerner y Lerner (2007) este indicador presenta un coeficiente poco aceptable (ver tabla 1).

Otro de los instrumentos que utilizan Lerner et al. (2004) para evaluar la C's, es el Self-Perception 
Tabla 1

Indicadores de medición de las cinco C's

\begin{tabular}{|c|c|c|c|}
\hline & & Reactivos & $\begin{array}{l}\text { Rango de Coeficientes } \\
\text { de alpha de Cronbach }\end{array}$ \\
\hline \multirow{2}{*}{ Confianza } & $\begin{array}{c}\text { Identidad positiva } \\
\text { (PSL-AB, Benson et al. 1998; Theokas et al. 2005) }\end{array}$ & 6 & .87 a $.88^{*}$ \\
\hline & $\begin{array}{c}\text { Autoestima } \\
\text { (SPPA, Harter, 1983) }\end{array}$ & 6 & .74 a $.82 *$ \\
\hline \multirow{4}{*}{ Competencia } & $\begin{array}{c}\text { Académica } \\
\text { (SPPA, Harter, 1983) }\end{array}$ & 6 & .78 a $.81 *$ \\
\hline & $\begin{array}{c}\text { Calificaciones } \\
\text { (PSL-AB, Benson et al. 1998; Theokas et al. 2005) }\end{array}$ & 1 & NA \\
\hline & $\begin{array}{c}\text { Social } \\
\text { (SPPA, Harter, 1983) }\end{array}$ & 6 & .76 a $.80^{*}$ \\
\hline & $\begin{array}{c}\text { Compromiso escolar } \\
\text { (PSL-AB, Benson et al. 1998; Theokas et al. 2005) }\end{array}$ & 4 & $.56^{* *}$ \\
\hline \multirow{4}{*}{ Carácter } & $\begin{array}{c}\text { Valores personales } \\
\text { (PSL-AB, Benson et al. 1998; Theokas et al. 2005) }\end{array}$ & 5 & .86 a $.87^{*}$ \\
\hline & $\begin{array}{c}\text { Consciencia social } \\
\text { (PSL-AB, Benson et al. 1998; Theokas et al. 2005) }\end{array}$ & 6 & .87 a $.89 *$ \\
\hline & $\begin{array}{l}\text { Valores de diversidad } \\
\text { (PSL-AB, Benson et al. 1998; Theokas et al, 2005) }\end{array}$ & 4 & .79 a $.81 *$ \\
\hline & $\begin{array}{l}\text { Valores y habilidades interpersonales } \\
\text { (PSL-AB, Benson et al. 1998; Theokas et al. 2005) }\end{array}$ & 6 & $.68 * *$ \\
\hline \multirow{5}{*}{ Cuidado } & $\begin{array}{c}\text { Simpatía: Desventaja } \\
\text { (ESS, Einsenberg et al. 1996) }\end{array}$ & 1 & \\
\hline & $\begin{array}{c}\text { Simpatía: Soledad } \\
\text { (ESS, Einsenberg et al. 1996) }\end{array}$ & 1 & \\
\hline & $\begin{array}{l}\text { Simpatía: Desdichado } \\
\text { (ESS, Einsenberg et al. 1996) }\end{array}$ & 1 & .83 a $.85^{*}$ \\
\hline & $\begin{array}{c}\text { Simpatía: Dolor } \\
\text { (ESS, Einsenberg et al. 1996) }\end{array}$ & 1 & \\
\hline & $\begin{array}{c}\text { Simpatía: Rechazo } \\
\text { (ESS, Einsenberg et al. 1996) }\end{array}$ & 1 & \\
\hline \multirow{4}{*}{ Conexión } & $\begin{array}{c}\text { Familia } \\
\text { (PSL-AB, Benson et al. 1998; Theokas et al. 2005) }\end{array}$ & 6 & .88 a $.90^{*}$ \\
\hline & $\begin{array}{c}\text { Escuela } \\
\text { (PSL-AB, Benson et al. 1998; Theokas et al. 2005) }\end{array}$ & 7 & .82 a $.84 *$ \\
\hline & $\begin{array}{c}\text { Comunidad } \\
\text { (PSL-AB, Benson et al. 1998; Theokas et al. 2005) }\end{array}$ & 5 & .90 a $.92 *$ \\
\hline & $\begin{array}{c}\text { Pares } \\
\text { (Peer Support Scale, Armsden \& Greenberg, 1987) }\end{array}$ & 4 & .95 a $.97 *$ \\
\hline
\end{tabular}

Nota. *Valores reportados en Bowers et al. 2010; **Jelicic et al. 2007; NA= No aplica.

Profile for Adolescents (SPPA) de Harter (1983), el cual fue diseñado originalmente para evaluar la percepción de competencia sobre cinco dominios específicos (competencia académica, social y física, apariencia física y conductas adecuadas) y una valoración global de autoestima en niños. Lerner et al. (2005) mencionan que realizaron una adecuación de la redacción de los reactivos para que estuvieran de acuerdo a la edad. De acuerdo a lo reportado por Bowers et al. (2010), los índices de confiabilidad reportados en las distintas mediciones respecto al SPPA fueron adecuados (ver tabla 1).

Para la evaluación del cuidado, Lerner et al. (2005) modifican cinco reactivos de la Eisenberg Sympathy Scale (ESS, Einsenberg, Fabes, \& Murphy, 1996), la cual busca evaluar el grado en que los adolescentes sienten pena por el sufrimiento de otros; cabe señalar, que algo que llama la atención es que en distintos artículos (Bowers et al. 2010; Jelicic et al., 2007; Lerner et al., 2005) reportan 
que cada uno de los reactivos utilizados evalúa un indicador de este constructo, por lo cual, no en todos los artículos refieren datos de la confiabilidad. El último instrumento que utilizan Lerner et al. (2005) es la Peer Support Scale de Armsden y Greenberg (1987), el cual sirve para evaluar el indicador de pares en la conexión y que de acuerdo a las distintas mediciones obtiene datos de confiabilidad aceptables.

Dado que el SQ de Lerner et al. (2005) es un instrumento diseñado para población adolescente norteamericana, Shek y Ma (2010) diseñaron un instrumento para evaluar el desarrollo positivo en población asiática. La Chinese Positive Youth Development Scale (CPYDS) es un a escala formada por 80 reactivos, divididos en 15 sub-escalas: vínculos, resiliencia, competencia social, conducta positiva, competencia emocional, cognitiva, conductual y moral, auto-determinación, autoeficacia, identidad positiva, creencias del futuro, participación y normas prosociales, y espiritualidad. Los autores reportan que estas sub-escalas se agrupan en cuatro grandes componentes: competencias cognitivo-conductuales, atributos sociales, identidad positiva y cualidades de desarrollo positivo general. Asimismo, reportan que el instrumento ha mostrado ser consistente en su estructura en diferentes muestras de adolescentes.

Por otro lado, Lopez, Yoder, Brisson, Lechuga-Pena y Jenson (2015) crearon el Bridge-PYD, el cual es un instrumento que busca evaluar el desarrollo positivo en niños y adolescentes desde el modelo de las 5 C's, el cual se conformó con 9 reactivos que evalúan la Competencia, 10 reactivos que miden la Confianza, 10 reactivos que miden la Conexión, 11 reactivos que evalúan la dimensión de Carácter y 7 reactivos más que conforman la dimensión de Conexión. Los hallazgos que reportan los autores indican que el Bridge-PYD es un instrumento relativamente fácil de administrar y que además obtuvieron indicadores adecuados de validez. Asimismo, los resultados mostraron un ajuste adecuado del modelo, específicamente se encontró que las cinco sub-escalas convergen para explicar un constructo de alto orden que es el PYD.

Como se puede apreciar existen diferentes instrumentos que se han desarrollado para evaluar el desarrollo positivo desde el modelo de las 5 C's. Sin embargo, como menciona Gelhof et al. (2015), los instrumentos deben ser diseñados para el contexto de la población que se evaluará y en México no existen hasta ahora instrumentos que trabajen desde este modelo, es por ello, que el objetivo de este estudio fue diseñar y evaluar psicométricamente un instrumento que evalúa el desarrollo positivo de adolescentes mexicanos.

\section{Materiales y Métodos}

\section{Diseño}

No experimental, transversal.

\section{Participantes}

Se seleccionó una muestra no probabilística de 400 adolescentes, estudiantes de cinco escuelas secundarias, cuatro privadas y una pública, de dos estados: de México y Guanajuato. El 57\% fueron hombres y el $43 \%$ mujeres, con un rango de edad de 11 a 16 años $(M=13.52, D E=0.89)$. El $15.8 \%$ de los participantes cursaban el primer grado, $48 \%$ segundo grado y $36.3 \%$ tercer grado. El único criterio de inclusión que se consideró fue que los adolescentes fueran estudiantes de las instituciones donde se obtuvo la información. Se obtuvo el consentimiento de los padres para administrar los instrumentos del estudio en el marco institucional.

\section{Instrumento}

Se diseñó un instrumento basado en la propuesta de Lerner et al. (2004), el cual se conforma de 59 reactivos que se agrupan en cinco escalas que evalúan las 5 C's: Competencia (13 reactivos), Cuidado (6 reactivos), Confianza (11 reactivos), Conexión (17 reactivos) y Carácter (12 reactivos). Todas las escalas fueron tipo Likert con cinco opciones de respuesta, para cuatro de las escalas las opciones fueron de Totalmente de acuerdo a Totalmente en desacuerdo, solo para la escala de Cuidado las opciones fueron de Nunca a Siempre. El desarrollo del instrumento se llevó a cabo por medio de la revisión de la literatura, específicamente para las escalas de Competencia y Confianza se utilizaron algunos reactivos de las escalas Harter (1983) y de Andrade (2013), por lo que se refiere a las otras tres escalas se diseñaron específicamente para este estudio. Ya con el instrumento elaborado se procedió a la validez de contenido por medio de jueces expertos en población adolescente y que además conocen la PYD. En función de las evaluaciones y sugerencias de los expertos se realizaron algunas modificaciones al instrumento original, en 
general fueron ajustes de redacción y de eliminar algunos reactivos.

\section{Procedimiento}

Se solicitó permiso a las autoridades de las instituciones educativas para poder llevar a cabo la aplicación del instrumento con sus estudiantes. $\mathrm{El}$ instrumento fue auto-aplicable y los estudiantes lo respondieron de manera grupal, en un tiempo aproximado de 20 minutos. A los participantes se les explicó el objetivo del estudio y el uso confidencial de la información. En dos de las cinco instituciones la aplicación se llevó a cabo pero el departamento de Psicología de la propia institución.

\section{Análisis de datos}

Para analizar la discriminación de los reactivos se realizaron: análisis de frecuencias, correlación ítem-total y grupos contrastados; el criterio que se siguió para que los reactivos no fueran eliminados de los análisis posteriores es que cumplieran con los criterios de al menos dos de los tres análisis realizados. Los resultados indicaron que todos los reactivos cumplieron de manera satisfactoria con al menos dos de los criterios, así que todos los reactivos fueron incluidos en los análisis posteriores.

Con el fin de determinar la dimensionalidad de los constructos, se llevaron a cabo análisis factoriales de componentes principales con rotación ortogonal para cada una de las escalas, el criterio que se siguió para considerar un reactivo dentro de un factor fue que presentara carga factorial mayor a 0.40 y sin una carga factorial similar en otro factor. Posteriormente, para determinar el número de factores que componían cada una de las escalas se consideró en primer lugar la claridad conceptual y que como mínimo, tuvieran tres reactivos; además, se obtuvo el Alpha de Cronbach para determinar la consistencia interna de cada factor.

\section{Resultados}

Respecto a los resultados de la primera escala que evalúa Competencia, los análisis arrojaron tres factores que explicaron el $54.23 \%$ de la varianza, sin embargo, debido a que en el tercer factor sólo quedaba un reactivo se eliminó y solo quedaron dos factores que explicaron el $50.93 \%$ de la varianza. Como se puede observar en la tabla 2, en el primer factor se agruparon siete reactivos que evalúan la percepción del adolescente sobre su competencia académica, en el segundo factor se agruparon tres reactivos respecto a la percepción de los adolescentes sobre la competencia social. La confiabilidad para el total de la escala de Competencia fue de $\alpha=.809$, lo cual indica una consistencia interna adecuada; al hacer el análisis por cada factor, los resultados mostraron una confiabilidad más alta en la competencia académica $(\alpha=$ .831) respecto a la competencia social $(\alpha=.638)$.

Tabla 2

Análisis Factorial y Consistencia Interna de la Escala Competencia

\begin{tabular}{lrr}
\hline \multicolumn{1}{c}{ Reactivos } & Peso Factorial \\
\cline { 2 - 3 } & Factor 1 & Factor 2 \\
\hline 1. Soy bueno para las tareas escolares. & .690 & .771 \\
3. Tengo buenas calificaciones & .543 & .655 \\
5. Me considero tan inteligente como la mayoría de mis compañeros. & .829 \\
7. Tengo la capacidad para comprender bien la mayor parte de las materias de la escuela & .708 \\
9. Suelo aprobar las materias con facilidad e incluso con buenas calificaciones & .664 \\
11. Estoy preparado y me considero capaz de conseguir logros académicos & .676 \\
13. Confío en mi capacidad académica. & .839 \\
6. Soy capaz de adaptarme a situaciones sociales desconocidas & .716 \\
10. En general, me es fácil hacer amigos & Alfa de Cronbach & .831 \\
12. Me considero importante para la mayoría de mis amigos & .638 \\
\hline
\end{tabular}


En cuanto a los resultados de la escala de Cuidado (ver tabla 3), los análisis indicaron que los seis reactivos se agruparon en un solo factor que explicó el $39.52 \%$ de la varianza; la consistencia interna de la escala se encontró en parámetros aceptables $(\alpha=.672)$.

Por lo que se refiere a la escala de Confianza, los hallazgos arrojaron dos factores que explicaron el $49.24 \%$ de la varianza. En el primer factor se agruparon seis reactivos que evalúan la percepción que tiene el joven de sus cualidades por lo cual se nombró identidad positiva. Para el segundo factor, se agruparon cinco reactivos que valoran la autoestima del adolescente (ver tabla 4). La consistencia interna de esta escala fue de $\alpha=.837$, lo cual indica una confiabilidad adecuada, asimismo, al realizar el análisis por cada uno de los factores se obtuvieron valores adecuados $(\alpha=.749$ para identidad positiva y $\alpha=.767$ para autoestima).
En los hallazgos de la escala de Conexión, como se puede observar en la tabla 5, los resultados indicaron que los reactivos se agruparon en dos factores que explicaron el $60.37 \%$ de la varianza. En el primer factor se agruparon nueve reactivos que evalúan el vínculo que se tiene con los pares, en el segundo factor se agruparon ocho reactivos que miden la conexión con los padres. Respecto a la consistencia interna de la escala total se obtuvo una $\alpha=.920$, lo cual indica una alta confiabilidad, este valor, se mantuvo de forma similar al realizar el análisis por cada uno de los factores $(\alpha=.903$ para conexión con pares y $\alpha=.919$ para conexión con padres).

Finalmente, para la escala de Carácter, los hallazgos arrojaron un solo factor que explicó el $39.27 \%$ de la varianza, conformado por 12 reactivos (ver tabla 6$)$, con una consistencia interna adecuada $(\alpha=.854)$.

Tabla 3

Análisis Factorial y Consistencia Interna de la Escala Cuidado

\begin{tabular}{|c|c|}
\hline Reactivos & $\begin{array}{c}\text { Peso } \\
\text { factorial }\end{array}$ \\
\hline 1.Me conmueve el dolor de otras personas & .550 \\
\hline 2. Me preocupo por los demás & .698 \\
\hline 3. Me molesta cuando se comete alguna injusticia & .599 \\
\hline 4. Tomo en cuenta los sentimientos de los demás & .736 \\
\hline 5. Cuando veo que alguien está en problemas me dan ganas de ayudarlo & .729 \\
\hline 6. Cuando un amigo o familiar atraviesa por momentos difíciles suelo darme cuenta sin que me lo diga & .388 \\
\hline 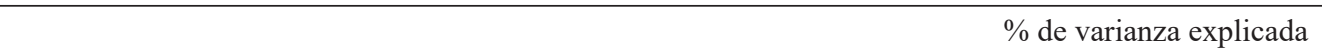 & 39.52 \\
\hline Alfa de Cronbach & .672 \\
\hline
\end{tabular}

Tabla 4

Análisis Factorial y Consistencia Interna de la Escala Confianza

\begin{tabular}{lcc}
\hline \multicolumn{1}{c}{ Reactivos } & Peso Factorial \\
\cline { 2 - 3 } & Factor 1 & Factor 2 \\
\hline 2. Puedo encontrar la manera de obtener lo que quiero a pesar de las dificultades & .570 \\
3. Considero que soy bueno para la mayoría de las cosas & .643 \\
4. Puedo resolver problemas difíciles si me esfuerzo lo suficiente & .590 \\
5. Reconozco mis cualidades & .500 \\
6. Manejo adecuadamente situaciones inesperadas & .710 \\
9. Gracias a mis cualidades puedo superar situaciones imprevistas & .695 & .708 \\
1.Me siento orgulloso de cómo soy & & .522 \\
7. Soy una persona valiosa & .786 \\
8. Me siento satisfecho conmigo mismo & & .802 \\
10. Me gusta mi forma de ser & & .509 \\
11. Considero que soy alguien que le cae bien a los demás & & 10.69 \\
\hline \% Varianza explicada & 38.54 & .767 \\
\hline
\end{tabular}


Tabla 5

Análisis Factorial y Consistencia Interna de la Escala Conexión

\begin{tabular}{|c|c|c|}
\hline \multirow{2}{*}{ Reactivos } & \multicolumn{2}{|c|}{ Peso factorial } \\
\hline & Factor 1 & Factor 2 \\
\hline 1.Valoramos nuestra amistad & .772 & \\
\hline 2.Nos aceptamos tal como somos & .680 & \\
\hline 3.Disfrutamos pasar tiempo juntos & .776 & \\
\hline 4. Nos escuchamos cuando alguno tiene la necesidad de hablar & .736 & \\
\hline 5.Sentimos la necesidad de estar en contacto & .720 & \\
\hline 6.Nos comprendemos, aunque no siempre estemos de acuerdo & .660 & \\
\hline 7. Nos tenemos cariño & .802 & \\
\hline 8. Nos apoyamos cuando alguno tiene problemas & .765 & \\
\hline 9. Respetamos nuestras formas de pensar & .654 & \\
\hline 1.Respetamos nuestros sentimientos & & .745 \\
\hline 2. Nos aceptamos tal como somos & & .759 \\
\hline 3. Nos comprendemos a pesar de que en ocasiones no estemos de acuerdo & & .777 \\
\hline 4. Nos prestamos suficiente atención & & .781 \\
\hline 5. Nos escuchamos cuando alguno tiene la necesidad de hablar & & .826 \\
\hline 6. Nos consideramos una familia unida & & .824 \\
\hline 7. Disfrutamos de nuestra compañía & & .758 \\
\hline 8. Sabemos que contamos el uno con el otro cuando sea necesario & & .722 \\
\hline$\%$ varianza explicada & 44.23 & 16.14 \\
\hline Alfa de Cronbach & .903 & .919 \\
\hline
\end{tabular}

Tabla 6

Análisis Factorial y Consistencia Interna de la Escala Carácter

\begin{tabular}{lc}
\hline \multicolumn{1}{c}{ Reactivos } & $\begin{array}{c}\text { Peso } \\
\text { Factorial }\end{array}$ \\
\hline 1. Soy honesto con los demás & .513 \\
2. Soy alguien que no se da por vencido fácilmente cuando lucha por algo justo & .545 \\
3. Para mi es importante respetar los valores y creencias de las personas sin importar que sean & .701 \\
diferentes & .686 \\
4. Soy leal con las personas cercanas a mi & .641 \\
5. Es importante para mi ser solidario con las personas que necesitan ayuda & .647 \\
6. Considero que es importante fomentar valores con los miembros de mi familia & .496 \\
7. Creo que es importante participar de manera activa en grupos sociales u organizaciones de mi & .630 \\
comunidad & .634 \\
8. Considero que debo luchar contra las injusticias sociales & .690 \\
9. Lo más importante para mí es respetar los valores y principios de la sociedad & .659 \\
10. Creo que debo comportarme de acuerdo a los principios en los que creo & .639 \\
11. Considero que debo asumir la responsabilidad de mis actos sin culpar a otros de mis errores & \% varianza explicada \\
12. Es preferible hacer lo correcto a pesar de las consecuencias & 39.27 \\
\hline
\end{tabular}




\section{Discusión y Conclusiones}

El propósito de la presente investigación fue desarrollar y evaluar las propiedades psicométricas de una instrumento para evaluar el desarrollo positivo de población adolescente de México desde el modelo de las 5 C's de Lerner et al. (2005), en su primera fase. Los hallazgos mostraron una adecuada estructura factorial de las cinco (Competencia, Cuidado, Confianza, Conexión y Carácter) escalas que conforman el instrumento, asimismo, los índices de confiabilidad de cada una de las escalas estuvieron dentro de los parámetros adecuados.

En el caso de la escala de Cuidado, los resultados fueron similares a los reportados en el instrumento de Lerner et al. (2005) ya que se mantienen las dos dimensiones: identidad positiva y autoestima, con confiabilidades adecuadas. No obstante los reactivos que se utilizaron para el diseño de esta escala fueron en función de escalas previas desarrolladas para población adolescente de México (Andrade, 2013). En cuanto a la escala de Competencia, el instrumento original de Lerner et al. (2005) propone que esta característica debe ser evaluada en cuatro áreas: académica, social, calificaciones y compromiso escolar, no obstante desde el diseño de esta escala se consideró que para evaluar esta característica sólo se tomarían el área académica y social, ya que las otras dos, forman parte del área académica. Los resultados del presente estudio mostraron una agrupación adecuada en las dos dimensiones que la conforman, sin embargo, en la competencia social se eliminaron tres reactivos dado que contribuían a la confiabilidad del instrumento, por lo cual, se sugiere que para aplicaciones posteriores se revise esta dimensión en busca de ampliar los indicadores que permitan evaluar esta dimensión. Cabe señalar que específicamente para el diseño de esta escala se adaptaron algunos de los reactivos de la escala de Harter (1983), lo cual pudiera explicar porque no funcionó tan bien como las otras, dado que la escala de dicho autor fue diseñada de inicio para población infantil y para otro contexto cultural.

Por lo que se refiere a la escala de Conexión, el instrumento original de Lerner et al. (2004) conformaba la evaluación de esta característica en cuatro áreas: familia, escuela, comunidad y pares. No obstante, para el presente estudio solo se evaluaron dos: pares y padres, ya que se con- sidero que eran las áreas más relevantes para la edad de los adolescentes de los cuales se obtuvo información. Los hallazgos en la presente investigación mostraron una claridad en la agrupación de reactivos y además, fue en estas dimensiones donde se obtuvieron los puntajes de confiablidad más altos y similares a los coeficientes reportados en Bowers et al. (2010).

En cuanto a la escala de Carácter, en el instrumento de Lerner et al. (2005) se evaluaron cuatro dimensiones: valores personales, consciencia social, valores de diversidad y valores y habilidades interpersonales. En el presente estudio sólo se consideró un factor general de esta característica que permite evaluar los valores personales y de consciencia social con los que cuenta el adolescente. Los resultados mostraron que efectivamente los reactivos que se incluyeron dentro de esta escala se agruparon en un solo factor con un coeficiente adecuado de confiabilidad, similar a lo reportado por Bowers et al. (2010). Por lo que se refiriere a la escala de Cuidado, en el instrumento de Lerner et al. (2004) se utilizan cinco reactivos de la escala de Einsenberg et al. (1996) donde en algunos casos los tratan como reactivos aislados y no reportan confiabilidad y en otros estudios si los reportan como una sola dimensión, para este estudio se diseñó una escala de seis reactivos que se agruparon en un solo factor, con una confiabilidad moderada, por lo cual, se recomienda para estudios posteriores revisar esta escala para ver si se pueden incluir algunos otros indicadores que permitan fortalecer más la consistencia interna de este constructo.

En términos generales, se puede decir que los resultados de este estudio muestran que el instrumento tiene indicadores psicométricos adecuados para evaluar el desarrollo positivo en población adolescente mexicana. Sin embargo, es importante dejar en claro que lo que aquí se reporta son datos de un análisis factorial exploratorio, por lo que, se recomienda aplicar el instrumento a una muestra más amplia para poder realizar análisis confirmatorios sobre la estructura del instrumento, con la finalidad de aportar información sobre la estructura y si ésta contribuye (desde las escalas) a evaluar el constructo desarrollo positivo.

Aún cuando los datos aquí presentados son de un primer análisis, se considera que este estudio aporta una herramienta que permite valorar desde el modelo de las 5 C's el desarrollo positivo en adolescentes, lo que puede servir para la valo- 
ración de programas de promoción del desarrollo saludable en adolescentes. Así como para llevar a cabo estudios longitudinales en los cuales se siga el proceso de cómo van cambiando estas características que describen a un adolescente con desarrollo positivo (Lerner et al. 2004), tomando siempre en consideración el momento en el que se encuentra el adolescente así como el contexto donde se está desarrollando lo cual es una parte fundamental de la PYD (Gelhof et al., 2015).

\section{Referencias}

Andrade, P. P. (2013). Informe Técnico del proyecto Desarrollo positivo del adolescente como protector de conductas de riesgo. México: UNAM.

Armsden, G. C., \& Greenberg, M. T. (1987). The inventory of parent and peer attachment: Individual differences and their relationship to psychological well-being in adolescence. Journal of Youth and Adolescence, 16(5), 427-454. doi: https://doi.org/10.1007/BF02202939

Benson, P. L., Leffert, N., Scales, P. C., \& Blyth, D. A. (2012). Beyond the "village" rhetoric: Creating healthy communities for children and adolescents. Applied Developmental Science, 16(1), 3-23. doi: https://doi.org/10. 1080/10888691.2012.642771

Bowers, E.P., Li, Y., Kiely, M.K., Brittain, A., Lerner, J.V., \& Lerner, R.M. (2010). The five Cs model of positive youth development: A longitudinal analysis of confirmatory factor structure and measurement invariance. Institute for Applied Research in Youth Development, Tufts University.

Eisenberg, N., Fabes, R. A., \& Murphy, B. C. (1996). Parents' reactions to children's negative emotions: Relations to children's social competence and comforting behavior. Child Development, 67(5), 2227-2247. doi: 10.1111/j.1467-8624.1996.tb01854.x

Geldhof, G.J., Bowers, E.P., Mueller, M. K., Napolitanto, C. M., Schmid, C. K., Walsh, K. J., Lerner, J. V., \& Lerner, R. (2015). The Five Cs Model of Positive Youth Development. En E. P. Bowers, G. J. Geldhof, S. K. Johnson, L. J. Hilliard, R. M. Hershberg, J. V. Lerner, \& R. M. Lerner (Eds.) Promotion Positive Youth Development. Lessons from the 4-h Study (pp. 161-186). USA: Springer.
Harter, S. (1983). Supplementary description of the Self-Perception Profile for Children: Revision of the Perceived Competence Scale for Children. Unpublished manuscript, University of Denver.

Heck, K. E., \& Subramaniam, A. (2009). Youth Development Frameworks. [Monograph]. Davis, CA. Recuperado de: http://4h.ucanr.edu/files/1224.pdf

Jelicic, H., Bobek, D. L., Phelps, E., Lerner, R. M., \& Lerner, J. V. (2007). Using positive youth development to predict contribution and risk behaviors in early adolescence: Findings from the first two waves of the 4-H Study of Positive Youth Development. International Journal of Behavioral Development, 31(3), 263-273. https:// doi.org/10.1177/0165025407076439

Lerner, R. M. (2002). Concepts and theories of human development. Mahwah, NJ: Lawrence Erlbaum.

Lerner, R. M. (2004). Liberty: Thriving and civic engagement among America's youth. Thousand Oaks, CA: Sage.

Lerner, R.M., Lerner, J.V., Almerigi, J.B., Theokas, C., Phelps, E., Gestsdottir, S... von Eye, A. (2005). Positive youth development, participation in community youth development programs, and community contributions of fifth-grade adolescents: Findings from the first wave of the 4-H study of positive youth development. Journal of Early Adolescence, 25, 1771. doi: https://doi.org/10.1177/0272431604272461

Lopez, A., Yoder, J. R., Brisson, D., Lechuga-Pena, S., \& Jenson, J. M. (2015). Development and validation of a positive youth development measure: The Bridge-Positive Youth Development. Research on Social Work Practice, 25(6), 726-736. doi: https://doi. org/10.1177/1049731514534899

Shek, D. T., \& Ma, C. M. (2010). Dimensionality of the Chinese positive youth development scale: confirmatory factor analyses. Social Indicators Research, 98(1), 4159. doi: https://doi.org/10.1007/s11205-009-9515-9

Theokas, C., Almerigi, J. B., Lerner, R. M., Dowling, E. M., Benson, P. L., Scales, P. C., \& von Eye, A. (2005). Conceptualizing and modeling individual and ecological asset components of thriving in early adolescence. The Journal of Early Adolescence, 25(1), 113-143. doi: https://doi.org/10.1177/0272431604272460 